\title{
Fabrication of porous calcium phosphate cements using gelatin as porogen
}

\author{
Jong-Hoon LEE, Hyoun-Ee KIM and Young-Hag KOH*, \\ Department of Materials Science and Engineering, Seoul National University, \\ Gwanak, 599, Gwanak-ro, Gwanak-gu, Seoul, 151-742, Korea \\ *Department of Dental Laboratory Science and Engineering, Korea University, \\ San, 1, Jeongreung-dong, Seongbuk-gu, Seoul, 136-703, Korea
}

\begin{abstract}
Porous calcium phosphate cements (CPCs) were fabricated using gelatin granules as the porogen. As the amount of gelatin was increased from 0 to $43 \mathrm{vol} \%$, the porosity notably increased from 3 to 47 vol\%, while the compressive strength decreased from 23 to $14 \mathrm{MPa}$; however, these values are still expected to be reasonably high for applications in bone tissue engineering. In addition, osteoblastic cells grew favorably on the surfaces of the CPCs prepared using gelatin of 11 and 43 vol\%, which contained well interconnected pores with a size of several hundreds of microns, indicating excellent biocompatibility of the porous CPC samples produced in this study.
\end{abstract}

(C2010 The Ceramic Society of Japan. All rights reserved.

Key-words : Porosity, Mechanical properties, Biomedical applications, Bone cements, Calcium phosphate

[Received August 11, 2009; Accepted October 15, 2009]

\section{Introduction}

Calcium phosphate cements (CPCs) have been proven to be highly effective in the repair of bone defects owing to their excellent osteoconductivity. ${ }^{1)-3)}$ In general, a paste prepared by mixing the CPC powder with an aqueous liquid is directly placed or injected into the bone defects, where it undergoes in-situ setting, being converted to low crystalline hydroxyapatite. Moreover, porous CPCs have recently received increasing interest since interconnected pores are expected to stimulate bone ingrowth inside the pores and ensure tight fixation. ${ }^{4)-6)}$

Porous CPCs have been previously produced by mixing watersoluble substances which act as a porogen, including mannitol, sucrose, and poly (lactic-co-glycolic acid) (PLGA), with a CPC paste, in which the porogen are expected to be degraded gradually in the body fluid and to create the pores required for favorable bone ingrowth. ${ }^{5-9)}$ In this study, we demonstrate the possibility of using gelatin granules as the porogen for creating interconnected pores in the CPC in-situ after implantation. Gelatin was selected since it is expected to be degraded readily in the body without inducing an inflammatory response. ${ }^{10)}$

\section{Experimental procedure}

Calcium phosphate cement (CPC) powder was prepared using a mixture of equimolar amounts of tetracalcium phosphate (TTCP) and dicalcium phosphate dihydrate (DCPD). Briefly, the TTCP powder was synthesized via the solid-state reaction between equimolar amounts of $\mathrm{CaHPO}_{4}$ (Alfa Aesar, USA) and $\mathrm{CaCO}_{3}$ (Sigma-Aldrich Ltd., USA) at $1500^{\circ} \mathrm{C}$ for $6 \mathrm{~h}$, followed by quenching to room-temperature and dry ball-milling. The high-purity DCPD powder was prepared by slowly raising the $\mathrm{pH}$ of a DCPD (Alfa Aesar, USA)-nitric acid solution to 3.5, followed by washing and drying. ${ }^{11)}$

Commercially available gelatin granules from porcine skin

Corresponding author: Y.-H. Koh; E-mail: kohyh@korea.ac.kr (type A, Sigma-Aldrich Ltd., USA) with a particle size range of $\sim 150-300 \mu \mathrm{m}$, measured by scanning electron microscopy (SEM, JSM-5600, JEOL Ltd.), were used as a porogen. The CPC pastes were prepared by mixing the CPC powder containing various amounts of gelatin granules $(0,11,21$, and $43 \mathrm{vol} \%)$ with a sodium phosphate-hydroxypropyl methylcellulose solution as the cement liquid at a powder-to-liquid ratio $(\mathrm{P} / \mathrm{L})$ of 2 . The prepared pastes were then transferred to a mold with dimensions of $\sim 7$ $\times 7 \times 14 \mathrm{~mm}$ and incubated in an oven with $100 \%$ relative humidity at $37^{\circ} \mathrm{C}$. The fully set samples were soaked for $2 \mathrm{~d}$ in simulated body fluid (SBF), prepared according to the method of Kokubo et al. ${ }^{12)}$ in order to remove the gelatin particles used as the porogen.

The microstructure and phase of the prepared samples after soaking in SBF, followed by washing and drying process were examined using scanning electron microscopy (SEM, JSM5600, JEOL Ltd.) and X-ray diffraction (XRD, M18XHF-SRA, Mac Science Company Ltd.), respectively. In order to evaluate the porosity and pore size, the porous samples were infiltrated with an epoxy resin (Spurrs epoxy, Polysciences Inc., Warrington, PA) and then cured at $70^{\circ} \mathrm{C}$ for $24 \mathrm{~h}$. Thereafter, the samples were ground and analyzed using SEM. The porosity and pore size of the samples were calculated from the SEM micrographs of the epoxy-filled samples. A minimum of five areas in each sample was used to obtain the average value.

For the compressive strength test, samples with dimensions of 6 $\times 6 \times 12 \mathrm{~mm}$ were loaded at a crosshead speed of $5 \mathrm{~mm} / \mathrm{min}$ using a screw driven load frame (Instron 5565, Instron Corp., Canton, MA, USA). Their compressive strengths were calculated from the compressive stress-strain curves. Seven samples were tested to obtain the average value along with its standard deviation.

The preliminary cellular assay was performed using a preosteoblast cell line (MC3T3-E1; ATCC, CRL-2593, USA). For the initial cell attachment, proliferation and differentiation tests, the cells were plated at a density of $5 \times 10^{4}$ cells $/ \mathrm{ml}$ and cultured in a humidified incubator with $5 \% \mathrm{CO}_{2}$ at $37^{\circ} \mathrm{C}$. Minimum essential medium ( $\alpha$-MEM: Welgene Co., Ltd., Seoul, Korea) 
supplemented with $10 \%$ FBS, $1 \%$ penicillin-streptomycin, 1, 3 and $10 \mathrm{mM} \beta$-glycerophosphate (GP), and $50 \mu \mathrm{g} / \mathrm{ml}$ ascorbic acid was used as the culturing medium. After culturing the cells on the samples for $3 \mathrm{~d}$, their growth morphology and proliferation were evaluated by SEM. In addition, the level of cell proliferation was measured after culturing for $3 \mathrm{~d}$ using an MTS assay (CellTiter 96 Aqueous One Solution, Promega, USA), which is considered to be an accurate tool for measuring the cell viability.

\section{Results and discussion}

The pore structures of the CPC samples after removing the gelatin particles by soaking them in the SBF solution for $2 \mathrm{~d}$ are shown in Fig. 1. Without the incorporation of the gelatin, no large pores but only relatively small closed pores were observed (Fig. 1(A)). On the other hand, a number of large pores with average sizes of several hundreds of microns were created by using the gelatin granules (Fig. 1(B)-(D)). It should be noted that the pore obtained should resemble the original shape and size of the gelatin used as the porogen. However, the sample became more porous with increasing the amount of gelatin. In addition, it was observed that, when 43 vol\% gelatin was added, all of the pores in the sample were infiltrated with an epoxy, indicating good interconnections between the pores (data not shown here.)

The porosity of the samples produced with the various
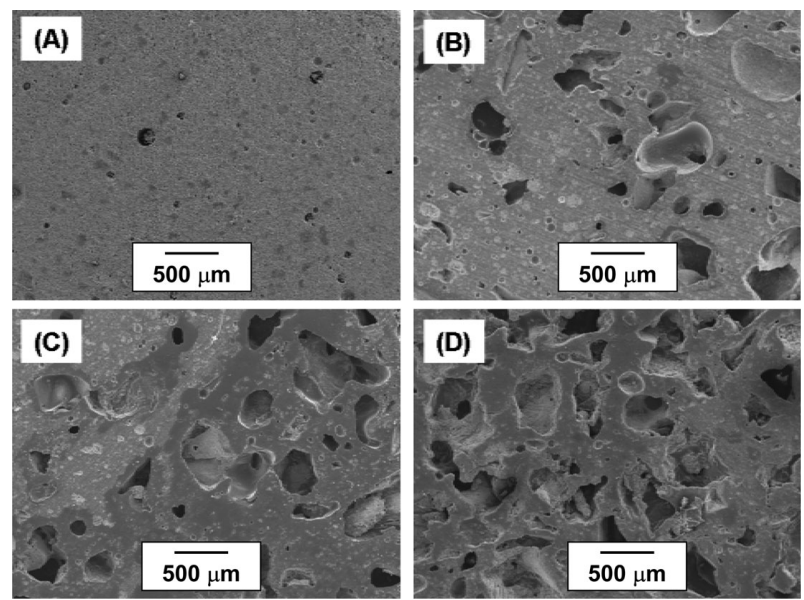

Fig. 1. SEM micrographs of the porous CPCs produced using various fractions of gelatin: (A) 0 , (B) 11 , (C) 21 , and (D) $43 \mathrm{vol} \%$.

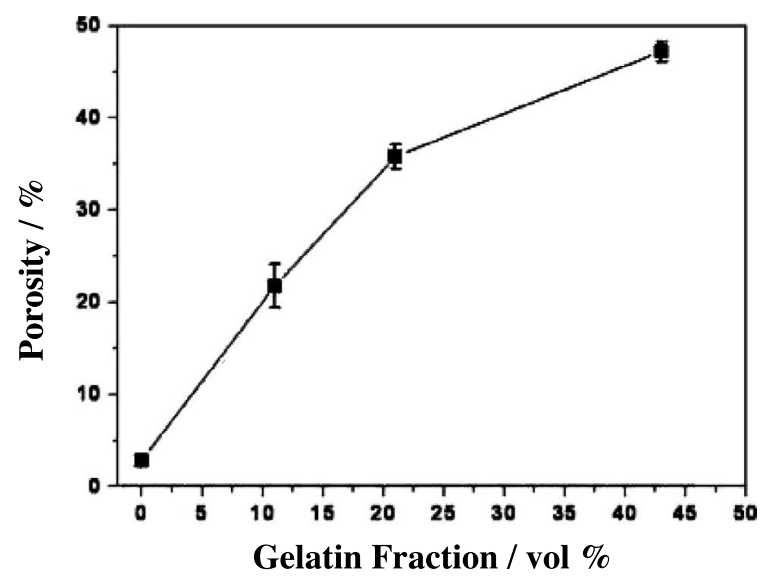

Fig. 2. Calculated porosity of the porous CPCs produced as a function of the as a function of the gelatin fraction. amounts of gelatin were measured, as shown in Fig. 2. As the amount of gelatin was increased from 0 to $43 \mathrm{vol} \%$, the porosity calculated on the basis of the SEM images notably increased from 3 to $47 \mathrm{vol} \%$. It should be noted that a higher fraction of gelatin would be expected to lead to a higher porosity; however, this would inevitably result in a considerable reduction in strength.

In order to evaluate the structural integrity of the porous CPCs, the compressive strength test was conducted. As the gelatin mass fraction was increased from 0 to $43 \mathrm{vol} \%$, the compressive strength decreased from 23 to $14 \mathrm{MPa}$, as shown in Fig. 3. The reduction in the compressive strength was apparently due to the increase in the porosity. ${ }^{7), 13)}$ However, these compressive strengths are comparable to those reported in the literature, ${ }^{5,7), 9)}$ indicating that the porous CPCs produced using gelatin as the porogen should find very useful applications in bone tissue engineering, in which interconnected pores are required for favorable bone ingrowth.

The crystalline phases of the samples were investigated by XRD analyses, as shown in Fig. 4. The sample without the addition of gelatin showed only crystalline hydroxyapatite (HA) peaks, implying the complete conversion to the HA phase via the setting reaction (Fig. 4(A)). However, when the gelatin granules were added as the porogen, several peaks of the TTCP phase were also observed (Fig. 4(B)-(D)). It has been demonstrated

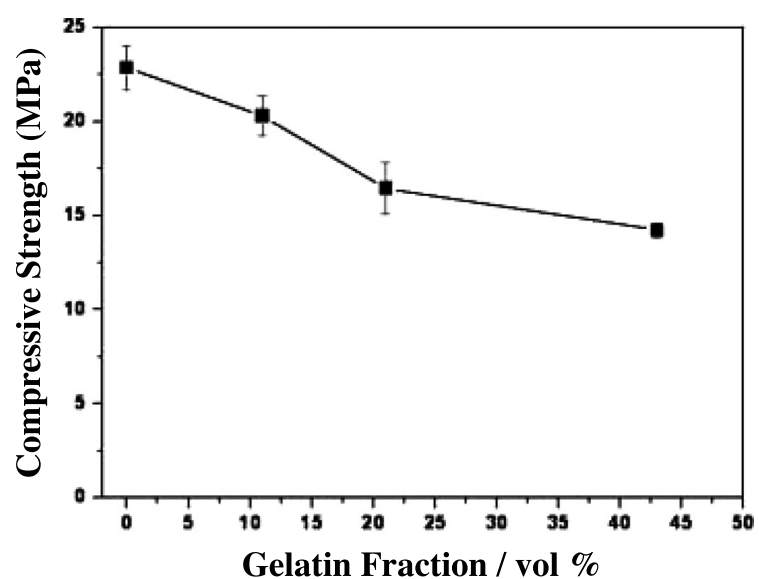

Fig. 3. Compressive strengths of the porous $\mathrm{CPC}$ samples as a function of the gelatin fraction.

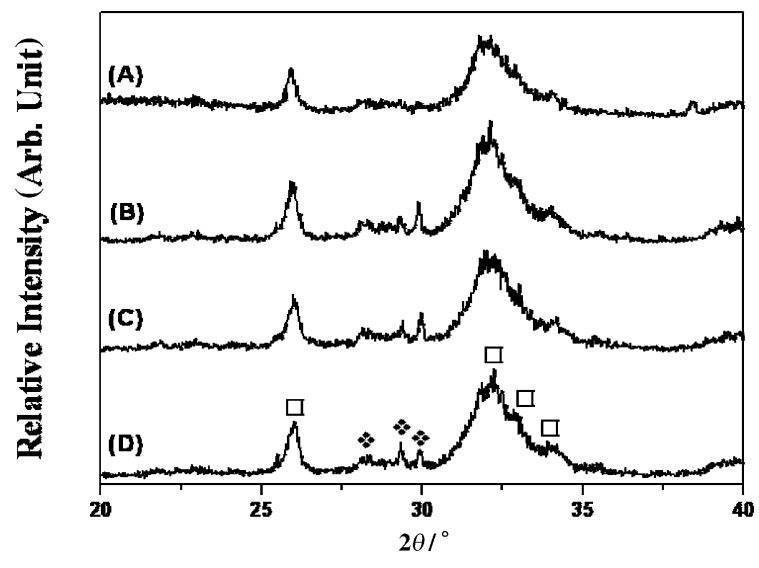

Fig. 4. XRD patterns of the porous $\mathrm{CPCs}$ produced using various mass fractions of gelatin: (A) 0, (B) 11, (C) 21, and (D) 43 vol\%. ( $\square$ : HA, : TTCP). 


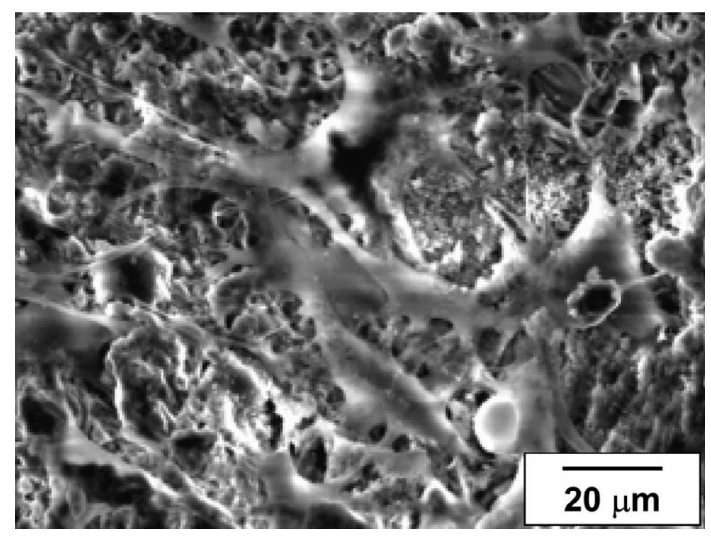

Fig. 5. Typical SEM micrograph of the porous CPC produced using a gelatin mass fraction of $43 \mathrm{vol} \%$, showing the cell attachment and proliferation.

that an aqueous gelatin solution can accelerate the setting reaction of CPC by interacting with mineral ions of the CPC. ${ }^{14)}$ However, in this study, the remaining TTCP phase was observed, which would be presumably due to the presence of gelatin in the form of solid granule. ${ }^{15}$ )

The possible influence of the remaining TTCP phase on the biological properties of the samples was examined in the preliminary in vitro tests using MC3T3-E1cells. All of the samples showed very similar behaviors, that is, the cells grew favorably on the cements with strong adhesion. The typical SEM micrograph of the sample prepared with a gelatin mass fraction of 43 vol\% is shown in Fig. 5, implying that the remaining TTCP phase would not be expected to seriously deteriorate the biological properties of the CPCS produced in this study.

In addition, the cell viability of the samples was quantified using the MTS method, as shown in Fig. 6. The cells on the porous CPC samples showed higher levels of cell viability after culturing for $3 \mathrm{~d}$, owing to the interconnections between the pores. However, a higher gelatin content was found to lead to a lower level of cell viability, presumably due to the presence of TTCP phase in the porous CPC samples. However, these values were comparable to that of the sample prepared without the addition of gelatin. These results indicate that the small amount of TTCP remaining is not detrimental to the biological properties of the CPCs produced in this study.

\section{Conclusions}

We fabricated porous calcium phosphate bone cements (CPCs) by adding gelatin granules up to $43 \mathrm{vol} \%$ as a porogen to CPC pastes. Pores with a size of several hundreds of microns were generated after soaking the samples in SBF solution for 2 $\mathrm{d}$. When the amount of gelatin was greater than $10 \mathrm{wt} \%$, most of pores were interconnected. The compressive strength of the samples decreased with increasing gelatin content; however, the lowest value was as high as $14 \mathrm{MPa}$ with a porosity of $47 \%$. In addition, it was observed that all of the prepared CPCs showed excellent biocompatibility in an in-vitro cell culture tests.

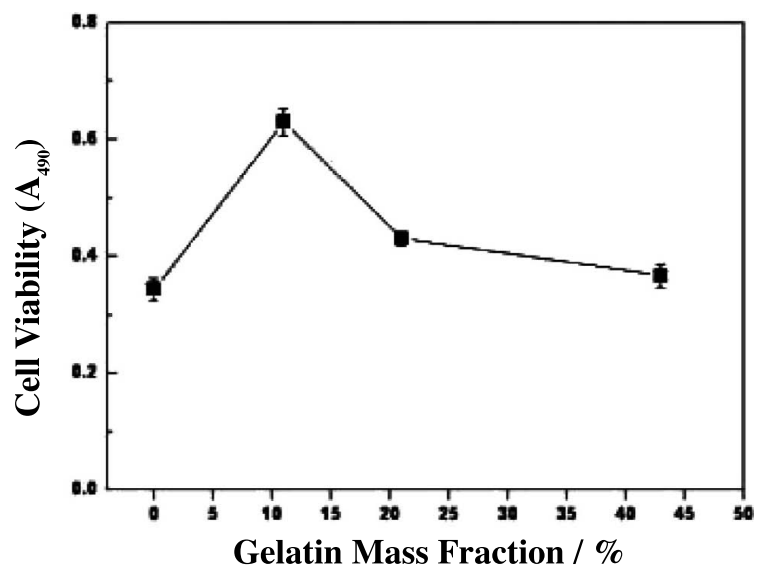

Fig. 6. Cell viability of the porous CPC samples as a function of the gelatin fraction.

Acknowledgments This research was supported by a grant from the Fundamental R\&D Program for Core Technology of Materials funded by the Ministry of Knowledge Economy, Republic of Korea.

\section{References}

1) B. R. Constantz, B. M. Barr, I. C. Ison, M. T. Fulmer, J. Baker, L. McKinney, S. B. Goodman, S. Gunasekaren, D. C. Delaney, J. Ross and R. D. Poser, J. Biomed. Mater. Res. A, 43, 451461 (1998).

2) Y. Miyamoto, K. Ishikawa, M. Takechi, T. Toh, T. Yuasa, M. Nagayama and K. Suzuki, J. Biomed. Mater. Res. A, 48, 3642 (1999).

3) D. Apelt, F. Theiss, A. O. El-Warrak, K. Zlinszky, R. BettschartWolfisberger, M. Bohner, S. Matter, J. A. Auer and B. von Rechenberg, Biomaterials, 25, 1439-1451 (2004).

4) R. P. del Real, J. G. Wolke, M. Vallet-Regi and J. A. Jansen, Biomaterials, 23, 3673-3680 (2002).

5) H. H. K. Xu, M. D. Weir, E. F. Burguera and A. M. Fraser, Biomaterials, 27, 4279-4287 (2006)

6) D. P. Link, J. van den Dolder, W. J. F. M. Jurgens, J. G. C. Wolke and J. A. Jansen, Biomaterials, 27, (2006)

7) H. H. K. Xu, J. B. Quinn, S. Takagi, L. C. Chow and F. C. Eichmiller, J. Biomed. Mater. Res., 57A, 457-466 (2001).

8) S. Takagi and L. C. Chow, J. Mater. Sci.: Mater. Med., 12, 135-139 (2001).

9) H. H. K. Xu, E. F. Burguera and L. E. Carey, Biomaterials, 28, 3786-3796 (2007).

10) S. Young, M. Wong, Y. Tabata and A. G. Mikos, J. Control. Release, 109, 256-274 (2005).

11) E. F. Burguera, F. Guitián and L. C. Chow, J. Biomed. Mater. Res., 71A, 275-282 (2004).

12) T. Kokubo, T. Yamamuro, L. L. Hench and J. Wilson, "CRC Handbook of Bioactive Ceramics," Boca Raton (FL): CRC Press (1990) pp. 41.

13) B. H. Yoon, Y. H. Koh, C. S. Park and H. E. Kim, J. Am Ceram. Soc., 90, 1744-1752 (2007).

14) A. Bigi, B. Bracci and S. Panzavolta, Biomaterials, 25, $2893-$ 2899 (2004).

15) Z. H. Pan, H. P. Cai, P. P. Jiang and Q. Y. Fan, J. Polymer. Res., 13, 323-327 (2006). 